\title{
A New Adaptive Controller for Performance Improvement of Automotive Suspension Systems with MR Dampers
}

\author{
Amir Khajepour and Ankur Agrawal \\ University of Waterloo
}

\begin{abstract}
A control algorithm is developed for active/semi-active suspensions which can provide more comfort and better handling simultaneously. A weighting parameter is tuned online which is derived from two components - slow and fast adaptation to assign weights to comfort and handling. After establishing through simulations that the proposed adaptive control algorithm can demonstrate a performance better than some controllers in prior-art, it is implemented on an actual vehicle (Cadillac STS) which is equipped with MR dampers and several sensors. The vehicle is tested on smooth and rough roads and over speed bumps.
\end{abstract}

CITATION: Khajepour, A. and Agrawal, A., "A New Adaptive Controller for Performance Improvement of Automotive Suspension Systems with MR Dampers," SAE Int. J. Passeng. Cars - Mech. Syst. 7(3):2014, doi:10.4271/2014-01-0052.

\section{INTRODUCTION}

The job of the suspension system in an automobile is dual: to provide a comfortable ride to the passengers by isolating them from the road irregularities, bumps and potholes, and to improve the road holding capacity of the vehicle thereby providing safety. The use of suspension systems in vehicles is not new. In fact, they have been in use since the cars were actually horse drawn carriages [1]. But still, active research has been prevalent for the development of new and better suspension systems. One major reason for this can be attributed to the fact that the two requirements of ride comfort and handling which the suspension is expected to fulfill are conflicting. Figure 1 shows this conflicting nature for different suspension parameters in terms of the RMS acceleration of the chassis (comfort) and RMS dynamic tire force (handling and safety) for some particular road and driving conditions. It can be seen that for better ride comfort (as in a Limousine), a softer suspension (low $\mathrm{k}$ and $\mathrm{d}$ ) is required but it leads to higher tire forces, hence, less safety. On the other hand, for better handling (as in a sports car), a stiffer suspension (high $\mathrm{k}$ and $\mathrm{d}$ ) is required but it makes the ride less comfortable. $A$ conventional suspension with a passive spring and damper is represented by a fixed point on this conflict diagram. Numerous efforts have been made by researchers to design a suspension system which caters to a wide range of performance requirements with as less compromise as possible by changing its properties during run-time like active and semi-active suspensions to give near optimal performance. Such suspensions with variable properties have led to the design of several control algorithms like skyhook, groundhook, clipped optimal, etc. which offer better results in different aspects of suspension performance.
An active suspension system consists of an actuator (electric or hydraulic) which can inject as well as dissipate power. Coupled with an appropriate controller, such a system can provide a performance far better than a typical semi-active system both in terms of comfort and handling. One such system developed by Bose Corporation [2] uses linear electromagnetic motors which can control each corner of the vehicle independently. Although the performance of this system is quite superior to that of a conventional suspension and Bose claims that the system can recover energy by driving the motors in generator mode, the system is yet to be integrated in a production vehicle, possibly due to high costs and power requirements. Most of the commercially available active suspensions as of now use hydraulic systems. For instance, the Dynamic Drive from BMW [] ] significantly reduces roll angle during cornering by using two active rotating hydraulic actuators. Mercedes-Benz has also developed an active suspension named as Active Body Control (ABC) [4] which consists of an active hydraulic cylinder mounted in series with the spring, which can rapidly move in the vertical direction by getting energized by a high pressure hydraulic pump.

A semi-active suspension system can change the damping characteristics during run-time but cannot provide a force input. It is represented on the conflict diagram in Figure 1 by a constant stiffness line. The level of damping can either be defined by the user through an instrumental panel or can be automatically controlled for that particular state of the vehicle by an on-board CPU that takes feedback from various sensors mounted on it. There are several ways through which the damping can be varied in a semi-active system. One popular product is MagneRide ${ }^{\mathrm{TM}}$ by BWI Group which uses Magneto- 
Rheological (MR) fluids [5]. An MR fluid has magnetically soft (easily, but temporarily magnetized) iron particles suspended in a synthetic hydrocarbon base. Application of magnetic field by the electromagnetic coil contained in the piston causes the particles to align into fibrous structures thereby increasing the viscosity. Hence, varying the magnetic flux in effect controls the viscosity of the damper. Another method of varying the damping is by changing the orifice size, as is done in the CDC ${ }^{\circledR}$-Continuous Damping Control by ZF [ 6 ]. The CDC has a proportional valve, which offers soft damping when the opening for the oil flow is expanded and firm damping when it is restricted.

Since variable dampers are practically and economically viable, it is worth investigating the possibilities of better control algorithms for semi-active suspensions. In this research, a new controller is proposed which is benchmarked against some well-accepted algorithms in prior-art, first through simulations and then by implementation on a fully instrumented Cadillac STS equipped with MR dampers.

\section{CONTROL ALGORITHMS IN PRIOR-ART}

A semi-active control algorithm varies the damping in order to obtain either better comfort, or handling, or both (with weights assigned to each). An adaptive control algorithm accounts for the variation in system parameters and the assignment of weights to the performance criteria. A two degree of freedom quarter car model as shown in Figure 2 will be considered throughout this research to define a suspension system with variable damping. Some of the widely popular control algorithms in literature are discussed below.

Skyhook control: Introduced by Karnopp et al. [7], it is one of the most popular comfort oriented control strategies. Originally developed for a single degree of freedom quarter-car model (no unsprung mass), it tries to emulate a fictitious damper $d_{\text {sky }}$ attached between the sprung mass and the stationary sky so that its movement is minimized thereby maximizing comfort. However, since it is practically realized by a damper mounted between the sprung and the sprung mass which can only apply force in the direction opposite to the relative velocity between them, the damping force is assumed to be zero when the passivity constraint is violated. Mathematically, it can be expressed as

$$
F_{d}= \begin{cases}-d_{s k y} \dot{z}_{s} & \text { for } \dot{z}_{s}\left(\dot{z}_{s}-\dot{z}_{u}\right) \geq 0 \\ 0 & \text { for } \dot{z}_{s}\left(\dot{z}_{s}-\dot{z}_{u}\right)<0\end{cases}
$$

The skyhook control strategy greatly attenuates the motion of the sprung mass. However, when implemented to a little more realistic two degree of freedom model, this technique leads to extreme vibrations of the unsprung mass (wheel hop) and high dynamic tire forces, which deteriorates vehicle stability.
Groundhook control: Analogous to the skyhook algorithm, the groundhook control algorithm [] ] tries to attenuate the dynamic tire force by emulating a fictitious damper $d_{g n d}$ attached between the unsprung mass and a static frame on the ground. Mathematically,

$$
F_{d}= \begin{cases}d_{g n d} \dot{z}_{u} & \text { for } \dot{z}_{u}\left(\dot{z}_{s}-\dot{z}_{u}\right) \leq 0 \\ 0 & \text { for } \dot{z}_{u}\left(\dot{z}_{s}-\dot{z}_{u}\right)>0\end{cases}
$$

Clipped optimal control: In this control scheme, first an optimal controller is designed using techniques like LQR or LQG, to generate the optimal control force $F_{a}$ which is assumed to take any arbitrary value [9], i.e., can act as an actuator as well as an energy dissipater. Then the passivity constraint is invoked to "clip" the force when it needs to inject power. It can be expressed mathematically as

$$
F_{d}= \begin{cases}0 & \text { if } F_{a}\left(\dot{z}_{s}-\dot{z}_{u}\right)>0 \\ F_{a} & \text { if } F_{a}\left(\dot{z}_{s}-\dot{z}_{u}\right) \leq 0\end{cases}
$$

where $F_{a}$ is the actuator force that would be optimally required if the system was fully active. It should be noted here that the term "optimal" is described in the performance index sense, which is defined by the designer as per requirement. The performance index might consist of cost on sprung mass movement, tire deflection, suspension deflection and input force, with weights assigned to each. The numerical value of the performance index, as such, has no physical significance.

\section{ADAPTIVE SEMI-ACTIVE SUSPENSION: MODELING, CONTROL AND SIMULATION RESULTS}

\section{Estimation}

Since comfort and handling are two conflicting criteria for suspension design, therefore, when designing a control algorithm for a semi-active suspension (or active suspension for that matter), it would be worthwhile to keep track of the two performance costs on-line and tune the weights assigned to the two criteria accordingly to obtain a balanced performance. Moreover, other variables like unsprung mass acceleration, suspension rattle velocity, etc. are also of interest for implementing the controller. All these quantities of interest must be attainable using commonly available and economical sensors in a vehicle. Since an accelerometer on the vehicle body (sprung mass) has become almost standard equipment on all modern cars due to its use in other systems as well (like ABS and ESC), measurement of comfort objective is relatively easy. Similarly, measurement of the suspension travel (relative displacement between sprung and unsprung mass) is also possible using sensors like a potentiometer or an LVDT. On the contrary, direct measurement of tire forces requires quite 
expensive sensors, which are impractical for a passenger vehicle. Hence, in this research, a simple filter based estimator has been used as shown in Figure 3 to estimate the required quantities by directly measuring two values: sprung mass acceleration $\ddot{z}_{S}$ and relative displacement between sprung and unsprung mass $z_{s}-z_{u}$ (suspension travel), the sensors for which are relatively economical and practical to mount.

This is a very simple approach for estimation. Although more complex estimators like Extended Kalman Filter (EKF) can provide more accurate results, it gives practically useful data for the proof of concept of the controller, as shown in later sections.

\section{Adaptive Semi-Active Suspension Control}

In this section, a control algorithm for semi-active suspensions will be developed. First, two different control schemes, one handling oriented and other comfort oriented are proposed. Then, the two are integrated by introducing a modification of the adaptive control structure presented in [10] to finally obtain a controller which is simple, economical to implement, and automatically distributes its priority between comfort and handling on-the-run as per the requirement.

From the equations of motion of Figure 2, it can be derived that the tire force would approach to zero (thereby providing better handling) for a damping force of

$$
F_{d}=-m_{u} \ddot{z}_{u}+k_{p}\left(z_{s}-z_{u}\right)
$$

However, since $F_{d}$ is a passive force, this rule has to be modified to accommodate the passivity constraint.

$$
F_{d}=\left\{\begin{array}{l}
-m_{u} \ddot{z}_{u}+k_{p}\left(z_{s}-z_{u}\right) \ldots \\
\ldots \text { for }\left(-m_{u} \ddot{z}_{u}+k_{p}\left(z_{s}-z_{u}\right)\right)\left(\dot{z}_{s}-\dot{z}_{u}\right) \leq 0 \\
0 \text { for }\left(-m_{u} \ddot{z}_{u}+k_{p}\left(z_{s}-z_{u}\right)\right)\left(\dot{z}_{s}-\dot{z}_{u}\right)>0
\end{array}\right.
$$

Similarly, the acceleration of the sprung mass would approach to zero (thereby providing better comfort) for a damping force of

$$
F_{d}=k_{p}\left(z_{s}-z_{u}\right)
$$

which basically becomes the R-S controller [11] after satisfying the passivity constraint.

The two control schemes mentioned above cater to their own specific performance objective only. Hence, if there exists a weighting parameter $\alpha \in[0,1]$ such that it defines the weights given to the two performance indices, and its value adapts itself online as per the requirement from driving conditions, then we have a very simple yet elegant controller for semiactive suspension systems.

$$
\begin{aligned}
F_{a d p t} & =\alpha\left(-m_{u} \ddot{z}_{u}+k_{p}\left(z_{s}-z_{u}\right)\right)+(1-\alpha)\left(k_{p}\left(z_{s}-z_{u}\right)\right) \\
\Rightarrow F_{a d p t} & =k_{p}\left(z_{s}-z_{u}\right)-\alpha m_{u} \ddot{z}_{u}
\end{aligned}
$$

The damping force is

$$
F_{d}= \begin{cases}F_{a d p t} & \text { for } F_{a d p t}\left(\dot{z}_{s}-\dot{z}_{u}\right) \leq 0 \\ 0 & \text { for } F_{a d p t}\left(\dot{z}_{s}-\dot{z}_{u}\right)>0\end{cases}
$$

One interesting point to be noted here is that without the passivity constraint, Eq. (7) can be used as a controller for a fully active suspension system as well.

Hence, for one extreme value of the weighting parameter $\alpha=$ 0 , the controller is purely comfort oriented, while for the other extreme value $\alpha=1$, the controller only focuses on handling. Now the problem has been reduced to appropriately adjusting the value of $\alpha$ for maximum performance improvement. An adaptive controller introduced in [12] had the basic idea that comfort is the objective, as long as the dynamic tire forces are within certain limits. The weighting parameter $\alpha$ varying between zero and one defines the level of this constraint violation. On similar lines but with some modifications to suit our requirements, an adaptation scheme for $\alpha$ will be developed in the next section.

\section{Calculation of Weighting Parameter a}

From the method described in Figure 3, the dynamic tire forces can be easily estimated from commonly available sensors, and thus, it can be constantly monitored for constraint violation. It is known that the RMS value is generally used to quantify a stochastic variable like tire force over time. However, during actual driving conditions, singular events like bumps and potholes suddenly change the tire forces, which might not appear in the RMS value that immediately. Hence, the absolute value also needs to be kept tracked of. The weighting parameter $\alpha$, therefore, is derived from two components: slow adaptation (from RMS value $F_{\text {tire, RMS }}$ ) and fast adaptation (from instantaneous absolute value $\left.\left|F_{\text {tire }}\right|\right)$.

Assuming stochastic road input and Gaussian probability density of the dynamic tire force, its standard deviation over a time period $T$ will be given by

$$
\sigma_{F_{\text {tire }}}=\sqrt{\frac{1}{T} \int_{0}^{T} F_{\text {tire }}^{2}(\tau) \mathrm{d} \tau}
$$


Since the dynamic tire force has zero mean, its standard deviation is the same as its RMS value, i.e., $F_{\text {tire,RMS }}=\sigma_{F_{\text {tire }}}$. For a normally distributed zero mean stochastic signal, the $6 \sigma$ rule states that nearly all of its values lie within the bounds of 6 standard deviations. Applying the $6 \sigma$ rule to dynamic tire force would mean that $F_{\text {tire }}$ should remain within the bounds of the static tire force $F_{\text {stat }}$ for most of the time $T$. In other words, the wheel almost never leaves contact with the ground. Mathematically, the constraint can be formulated as

$$
F_{\text {tire }, \text { RMS }} \leq \frac{F_{\text {stat }}}{6}
$$

where the static wheel load $F_{\text {stat }}=\left(m_{s}+m_{u}\right) g$.

To also take into account the variation of $F_{\text {tire }}$ due to singular events which might not reflect in its RMS value immediately, another constraint can be defined which takes into consideration its instantaneous value. It completely depends on the controller designer that what value of the ratio $F_{\text {tire }} / F_{\text {stat }}$ is deemed permissible. Clearly, $F_{\text {tire }} / F_{\text {stat }} \geq-1$, with the extreme value of -1 indicating that the tire has just left contact with the ground. However, it would be too late if the controller waits for the ratio to become -1 before it changes the weighting parameter. Some buffer region is needed during which the controller can act and bring down the dynamic tire force. As an intelligent guess, a threshold of $-80 \%$ is selected for the ratio of dynamic to static tire force. Mathematically

$$
F_{\text {tire }}+0.8 F_{\text {stat }} \geq 0 \Rightarrow F_{\text {tire }} \geq-0.8 F_{\text {stat }}
$$

It should be noted here that only negative value of $F_{\text {tire }}$ is responsible for leaving contact with the ground. However, since tire has very low damping, a high positive value of $F_{\text {tire }}$ would soon be followed by a high negative value. To avoid unnecessary chatter in the controller, the absolute value $\left|F_{\text {tire }}\right|$ can be used.

$$
\left|F_{\text {tire }}\right| \leq 0.8 F_{\text {stat }}
$$

With the two constraint equations defined for slow and fast adaptation, a way can be developed to quantify the level of constraint violation. The adaptation structure is shown in Figure 4, where the input is the dynamic tire force $F_{\text {tire }}$ (which will be the estimated tire force $\hat{F}_{\text {tire }}$ in practice) and output is the weighting parameter $\alpha$.

The upper branch of the structure is responsible for fast adaptation. The ratio of dynamic to static tire force is input to a heuristic function $h(u)$ (Figure 5) which increases steeply from zero to one for input greater than 0.8 . The selection of heuristic function $h(u)$ is also based on intuitive guess. Since the absolute value of $F_{\text {tire }}$ is considered, $h(u)$ is symmetric about $y$-axis. The output from $h(u)$ is error $e_{f}$ which is then passed through a low pass filter with time constant $\tau_{f}$ to reduce the chatter and rapid variations in fast adaptation parameter $\alpha_{f}$. The lower branch of the structure outputs the slow adaptation parameter $\alpha_{s}$. The amount by which the RMS of the ratio of static to dynamic tire force deviates from the predefined threshold of $1 / 6$, designated as slow adaptation error $e_{s}$, is integrated with output limited between zero and one. Finally, the two parameters from slow and fast adaptation are added, and the minimum of the sum $\alpha_{s}+\alpha_{f}$ and unity gives the final value of the weighting parameter $\alpha$.

\section{Simulation Results}

A two degree-of-freedom quarter car model was modeled in MapleSim and simulated with different road profiles (rough, smooth, bump) as input (modeled in time domain as described in Appendix A and with different controllers. Assuming that the damping force can be varied to any arbitrary value within some bounds and all the required variables are either measured or estimated, the proposed adaptive controller was then benchmarked against passive damping, skyhook and groundhook controllers. A passive damping of $1500 \mathrm{Ns} / \mathrm{m}$ has been selected which provides a really good compromise between comfort and handling for quarter car parameters shown in Table 1, which are very close to that of the front-left suspension of a Cadillac STS (used for experimental verification in the next section).

Table 1. Quarter-car parameters used in simulation of different controllers

\begin{tabular}{|c|l|l|}
\hline Parameter & Description & Value \\
\hline$m_{s}$ & Sprung mass & $493 \mathrm{~kg}$ \\
\hline$m_{u}$ & Unsprung mass & $62 \mathrm{~kg}$ \\
\hline$k_{p}$ & Spring stiffness & $35600 \mathrm{~N} / \mathrm{m}$ \\
\hline$k_{t}$ & Tire stiffness & $277000 \mathrm{~N} / \mathrm{m}$ \\
\hline
\end{tabular}

Figures $6, \underline{7}$, and $\underline{8}$ show the simulation results for a transitional road input, where initially, the road profile is smooth (type-A), but switches to rough (type-C) at $t=20 \mathrm{~s}$, the total simulation time being $40 \mathrm{~s}$. The power spectral density of the acceleration of the sprung mass in the frequency band of maximum human sensitivity is shown in Figure 6 as an index of comfort. It is seen that over the expected result of skyhook controller being better and groundhook controller being worse than a passive system in improving comfort, the proposed adaptive algorithm shows a performance ranging from close to slightly better than the skyhook. A peak is discernible in the $1-2 \mathrm{~Hz}$ region which is clearly the resonant frequency of the sprung mass. The new controller seems to do a quite good job in reducing this peak as well.

It might occur to the reader that the difference in the performance of skyhook and proposed controller is not significant enough. However, the actual strength of adaptive controller can be seen in Figure 7, which compares the running RMS of the tire force with different controllers. While the skyhook controller leads to exceedingly high dynamic tire force, 
the adaptive controller shows handling very closely similar to that of the groundhook controller, which, however, is not good in providing comfort. Hence, the true efficacy of the proposed adaptive controller is providing a better compromise between comfort and handling than a passive system. The core of this controller are the adaptation parameters and Figure 8 shows how these parameters vary for the given case of smooth to rough road conditions. The fast adaptation parameter $\alpha_{f}$ is almost zero throughout, except for a small peak at $t=20 \mathrm{~s}$ where the road type changes abruptly. The slow adaptation parameter $\alpha_{s}$, on the other hand, is zero on smooth road as the RMS of dynamic tire force is quite low and does not violate the $6 \sigma$ condition described in the last section. It is when the road becomes rough that the tire force starts hitting bounds resulting in slow adaption error $e_{s}$ and thereby a positive slow adaptation parameter $\alpha_{s}$. Since the running RMS of the dynamic tire force keeps increasing from $t=20 \mathrm{~s}$ to $t=40 \mathrm{~s}$, so does $\alpha_{\mathrm{s}}$. The final weighting parameter $\alpha$ being the sum of the slow and fast adaptation parameters is almost the same as $\alpha_{s}$ in this case.

As mentioned before, the modeled quarter car system was also simulated with a bump as the road input. Figures $9, \underline{10}$ and $\underline{11}$ show the results for a bump of height $6 \mathrm{~cm}$, width $1.5 \mathrm{~m}$ and vehicle speed $10 \mathrm{~m} / \mathrm{s}$; quite similar to that shown in Figure B1. The bump starts at $t=0.5 \mathrm{~s}$. Since a bump is a transient event, the instantaneous values for chassis acceleration and tire force are generally of interest (and significance) than their RMS values. The variation of the acceleration of the sprung mass with time is plotted in Figure 9 for the same four controllers in action as described before. Again, with the proposed adaptive controller, the comfort is seen to be better than the passive system and very close to the skyhook controller in terms of peak in sprung mass' acceleration. The adaptive controller also manages to reduce the oscillations in $\ddot{z}_{s}$ a few moments after crossing the bump, unlike other controllers. This is due to its ability to change the weighting parameter online.

Even after providing a comfortable pass over the bump, the proposed adaptive controller does not lead to high fluctuations in dynamic tire force like those with skyhook controller. This is quite crucial during normal driving conditions. When a vehicle passes over a bump at medium to high speeds, the tire might leave contact with the ground as observed in the results in Figure 10. If a driver tends to apply brakes or a steering input in this time duration, the normal force between the ground and tire would not be sufficient enough to produce a braking or cornering effect. Hence, for a controlled handling and ride safety, $F_{\text {tire }}$ should be within bounds as is successfully done with the adaptive controller. It should be noted that a phase difference between the different plots has been intentionally added for distinctive clarity.

In the case of a bump, the fast adaptation parameter $\alpha_{f}$ plays the major role (Figure 11). As the absolute value of $F_{\text {tire }}$ crosses the threshold of 0.8 times the static tire force, the fast adaptation error $e_{f}$ rises sharply as output from the heuristic function $h(u)$, which effectively generates high value of $\alpha_{f}$. As soon as $F_{\text {tire }}$ is brought under control, there is no critical requirement of adaptation parameters and they approach back to zero.

Now that it has been established from theoretical simulations that an efficient adaptive controller has been designed, it must be validated through actual practical implementation. In the next section, the controller will be implemented on the MR dampers of a Cadillac STS and acquired data will be analyzed.

\section{EXPERIMENTAL VALIDATION}

Before discussing the performance of the controller in experiments, this section first describes in detail the experimental setup and implementation of theory in practice. Then the MR damper installed in the vehicle is characterized. Finally, the proposed controller's performance is evaluated by performing road tests with different controllers.

\section{Description of the Experimental Setup}

All the experiments in this research are performed on a fully instrumented Cadillac STS at the Mechatronic Vehicle Systems Lab., University of Waterloo. This vehicle was factory-equipped with MR dampers at all the four suspension corners, and for the purpose of this research, the front-left damper was hacked into by disconnecting the original connector and mounting a custom connector using which the current supplied to the damper can be varied as required. Since the vehicle is extensively used at the aforementioned laboratory for various research purposes, it is installed with several sensors, data acquisition boards, processor, etc. However, in this document, the hardware relevant to this research only will be described.

A six-axis inertial navigation system (Datron Technology) can measure the linear acceleration and velocity of the vehicle along all three axes, i.e., longitudinal, lateral and vertical; and the three rotational movements, i.e., roll, pitch and yaw. Out of these six degree of freedoms, only heave (vertical), roll and pitch are affected by the suspension system, and hence were recorded. The vehicle is also equipped with load sensors at the wheels which can measure forces and moments along all three axes at the contact patch, and for the purpose of studying the suspension system, only the vertical tire force was recorded. To measure the suspension travel, a string potentiometer was mounted between the chassis and the suspension control-arm. A dSpace AutoBox mounted with an $1 / O$ and a processor board is installed in the trunk of the car through which sensor data can be recorded, as well as a required PWM signal can be generated. A servo drive with current operation mode (AZ20A8DDC from Advanced Motion Controls) takes a PWM signal as input and supplies amplified current proportional to the signal's duty-cycle to the coils of MR damper. The structure of this experimental setup has been depicted in Figure 12. The signal from the wheel load sensor is shown with a dashed line as it is supposed to be used only for verification; the controller actually works on estimated value of tire force. 
Since most of the formulations till now were performed on a quarter car model which required the acceleration of the (quarter) sprung mass, but in the experimental setup only a single IMU is mounted on the vehicle, the acceleration of the front-left corner's sprung mass can be approximated from the vertical acceleration of the total vehicle's sprung mass and roll and pitch acceleration about its center of gravity assuming small angular movements. In an slightly modified SAE coordinate system [13] where the origin is at the CoG of the sprung mass (or chassis), the corner acceleration can be given by

$$
\ddot{z}_{s_{L F}}=\ddot{z}_{s_{C o G}}-X_{F} \ddot{\beta}-Y_{L} \ddot{\alpha}
$$

where $Y_{L}$ and $X_{L}$ are respectively the distances along the $Y$-axis and $X$-axis from the CoG of the chassis to the front-left tire contact patch as shown in Figure 13.

\section{Modeling the Characteristics of the MR Damper}

Before implementing any controller on the Cadillac STS, it is first necessary to have a characterization of the MR damper installed in it, which would comprise of a relation between the input current, the suspension rattle speed and the produced damping force. This would modify the controller such that it outputs a current $i$ instead of a damping force $F_{d}$, which is actually how it can be implemented on the vehicle.

The characterization of the MR damper was done on the vehicle itself without separately testing it. For different currents through the damper ranging from $0 \mathrm{~A}$ to $4.2 \mathrm{~A}$, harsh pitching maneuvers in a straight line were performed for each current to obtain large variations in suspension speed. The damping force was estimated from the equation of motion of the unsprung mass (which makes it somewhat independent from the dynamics of the other three corners).

$$
F_{d}=-m_{u} \ddot{z}_{u}+k_{p}\left(z_{s}-z_{u}\right)-k_{t}\left(z_{u}-z_{r}\right)
$$

To obtain the final characteristic of the damper, the local extreme (peak) values of suspension velocity and damping force were considered for each current value and a smooth, shape-preserving curve was fit through the data points. Figure 14 shows the obtained relation as a two dimensional map.

However, for implementing in a controller, an inverse mapping is required which gives a current value for a given damping force and suspension speed. The inverse mapping obtained from the damper characteristic is depicted in Figure 15. If the required damping force is in the same direction as the suspension velocity, or if the required damping force is less than what is achievable by the minimum current (zero) at a given suspension velocity, the mapping outputs zero current. The maximum current has been limited at $4.2 \mathrm{~A}$, so if an arbitrarily high damping force is required at a very low suspension velocity, the current output is $4.2 \mathrm{~A}$.

\section{Implementation of Controllers and Comparison of Results}

With the damper characteristics at hand and the estimator validated, the controllers could actually be implemented on the vehicle. Similar to the simulations, tests were performed on a track with smooth to rough (gravel) profile transition at a forward speed of $45 \mathrm{~km} / \mathrm{h}$, and over a speed bump at $40 \mathrm{~km} / \mathrm{h}$. The test conditions are shown in Figures 16, 17, $\underline{18}$, and 19 in the form of photographs of the roads and forward velocity of the vehicle (obtained from the IMU) for the four runs. In the satellite image of the test track, the smooth road and gravel area are distinguishable due to color contrast. It was tried to keep the vehicle speed as constant as possible around $45 \mathrm{~km} / \mathrm{h}$, but slight variations could not be avoided in that rough ride. Due to limited length of this patch of road, the test results have been cropped to span $t=1 \mathrm{~s}$ to $t=17 \mathrm{~s}$, duration during which the controllers were actually in action. It was easier to keep the speed almost constant at $40 \mathrm{~km} / \mathrm{h}$ on the bump while driving over it, as the test duration was quite short and the road was otherwise smooth. The same criteria of PSD of the acceleration of the sprung mass (front-left corner in this case) and running RMS of the dynamic tire force is used for comparing the performance of different controllers, just as in simulations. To emulate a passive damper, a constant current of 2 A was supplied to the MR damper.

Figures 20, $\underline{21}$ and $\underline{22}$ show the results for the smooth to gravel transitional track. The PSD of the sprung mass acceleration with the proposed adaptive controller was obtained to be lower than the passive and skyhook for most of the frequencies between $2-8 \mathrm{~Hz}$, implying greater comfort. It is also seen that Groundhook controller is the worst in providing comfort. The adaptive controller also shows better handling compared with passive and groundhook controller in terms of the running RMS of the tire force, while skyhook controller is worst in controlling dynamic tire force. Similar to the trend seen in the simulation results, the final weighting parameter $\alpha$ constitutes mostly of slow adaptation parameter $\alpha_{s}$ which increases gradually to account for the rough road and hence higher tire force. A peak seen in the fast adaptation parameter $\alpha_{f}$ was probably due to a rock in the gravel area.

Results from a test by running the vehicle over a bump at $40 \mathrm{~km} / \mathrm{h}$ are presented in Figures 23, 24, $\underline{25}$ and $\underline{26}$. A slight phase delay has been added in the first two plots for distinctive clarity. The peak value of corner's vertical acceleration with adaptive controller is almost equal to that obtained from skyhook controller, which is more comfortable than the passive suspension. The proposed controller also shows slightly less peak in the dynamic tire force compared with the groundhook controller, and substantially better than the passive suspension. Hence, overall, the adaptive controller gives a performance with less compromise between comfort and handling. Since the bump was on a relatively smooth road, there is no variation in the slow adaptation parameter $\alpha_{s}$. The fast adaptation parameter $\alpha_{f}$, however, has huge peaks during the time span of the bump. It is clipped at its highest value of 
one to obtain the final weighting parameter $\alpha$. The variation of current with time can be seen in Figure 26. Although there is no direct relation between $\alpha$ and the supplied current because the current depends on the required damping force as well as the suspension speed, however, it is somewhat intuitive that reducing the dynamic tire force would require more damping, and hence, more current. Therefore, some saturated current peaks can be seen concurrent with the peaks in $\alpha$.

\section{CONCLUSIONS AND FUTURE WORK}

A controller was designed for active/semi-active suspension systems which had a weighting parameter $\alpha \in[0,1]$ such that at one end $(\alpha=0)$, the controller outputs a force which provides maximum comfort while at the other end $(\alpha=1)$, it is purely handling oriented. For semi-active systems, the force is set to be zero whenever it violates the passivity constraint, i.e., power needs to be injected. The weighting parameter $\alpha$ is adjusted online depending on the value of the dynamic tire force, both RMS and absolute. The controller is practically economical to implement as it only uses sensors to measure the acceleration of the sprung mass and the suspension travel. All other variables of interest are estimated from these two measurements. After proving via simulations, experimental implementation on one corner (front-left) of a fully instrumented Cadillac STS equipped with MR dampers was performed for two driving scenarios: at $45 \mathrm{~km} / \mathrm{h}$ on a road with smooth to rough transition and over a road bump at $40 \mathrm{~km} / \mathrm{h}$. For both the cases, the controller could adapt itself to the driving conditions and provided ride comfort and handling better or similar to the skyhook and groundhook controller respectively. While the skyhook controller led to very high dynamic tire force and the groundhook controller was not good in providing comfort, the proposed adaptive controller demonstrated a performance better than the passive system for both the criteria and both driving scenarios.

After the proof of concept on a single corner of the vehicle, it would be worthwhile to implement the controller on all four corners of the vehicle. For better results, the controller structure would need to be modified for a 7-dof full-car model from a 2-dof quarter-car one. As the sprung mass in this model will have three degrees of freedom, i.e., roll, pitch and vertical heave, the controller can also be modified to take into account roll and pitch stability of the vehicle during harsh cornering and accelerating (or decelerating) maneuvers respectively.

\section{REFERENCES}

1. Adams, W.B., "English Pleasure Carriages", C. Knight \& Co., 1837

2. Bose Corporation, "Bose Suspension System", http://www.bose. com/controller?url=/automotive/bose_suspension/the system.jsp Oct 2013.

3. BMW, "Dynamic Drive", http://www.bmw.com/com/en/ newvehicles/6series/coupe/2007/allfacts/engine dynamicdrive. html, Oct 2013.
4. Daimler AG, "Active Body Control" http://www.cubiccapacity.com/ wp-content/uploads/2008/12/prescan2.JPG, 2008

5. BWI Group, "Magneride", http://www.bwigroup.com/en/pshow. php?pid=22, Oct 2013.

6. ZF Friedrichshafen AG, "Continuous Damping Control", http://www. zf.com/corporate/en/products/product range/cars/cars cdc.shtml, Oct 2013.

7. Karnopp, D., "Theoretical limitations in active vehicle suspensions", Vehicle System Dynamics, 15(1):41-54, 1986, doi:10.1080/00423118608968839.

8. Valek, M., Novk, M., Ika, Z., and Vaculn, O., "Extended ground-hook - new concept of semi-active control of truck's suspension", Vehicle System Dynamics, 27(5-6):289-303, 1997, doi:10.1080/00423119708969333

9. Tseng, H.E., and Hedrick, J.K., "Semi-active control laws - optimal and sub-optimal", Vehicle System Dynamics, 23(7):545-569, 1994, doi:10.1080/00423119408969074

10. Koch, G., Diepold, K., and Lohmann, B., "Multi-objective road adaptive control of an active suspension system", Motion and Vibration Control, Springer Netherlands, 189-200, 2009, doi:10.1007/978-1-4020-9438-5 19.

11. Rakheja, S., and Sankar, S., "Vibration and shock isolation performance of a semi-active 'on-off' damper", American Society of Mechanical Engineers, 1985.

12. Venhovens, P.J.T., "Development and implementation of adaptive semi-active suspension control", Vehicle System Dynamics, 23(3):211-235, 1994, doi:10.1080/00423119408969057.

13. SAE International Surface Vehicle Information Report, "Sign Convention for Vehicle Crash Testing," SAE Standard J1733, Issued Dec. 1994

14. ISO 8608:1995. Mechanical vibration-road surface profiles: Reporting of measured data. International Organization for Standardization, Geneva, Switzerland, Tech. Rep., 1995.

15. Fialho, I.J., and Balas, G.J., "Design of nonlinear controllers for active vehicle suspensions using parameter-varying control synthesis", Vehicle System Dynamics, 33(5):351-370, 2000, doi:10.1076/0042-3114(200005)33:5;1-Q;FT351.

\section{ACKNOWLEDGMENTS}

The authors would like to thank Maplesoft Corporation for their technical and financial support.

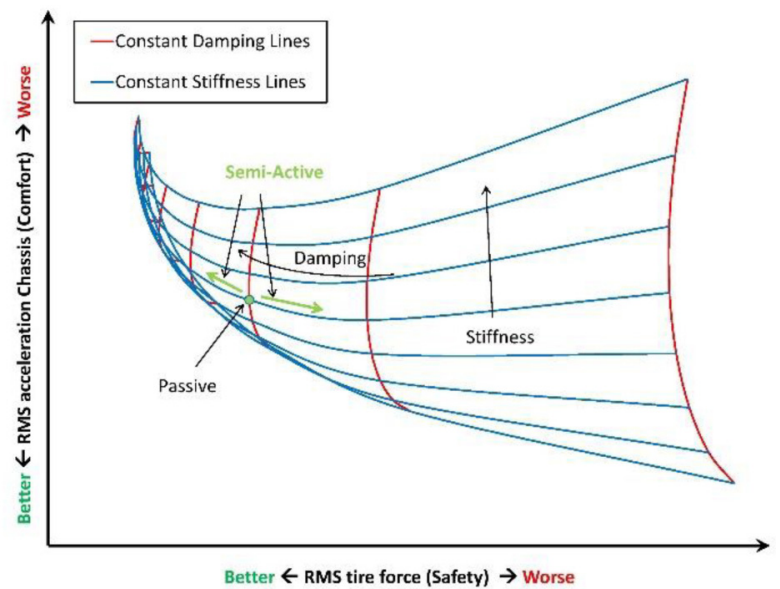

Figure 1. Conflict between safety and ride comfort (for some particular road and driving conditions). 


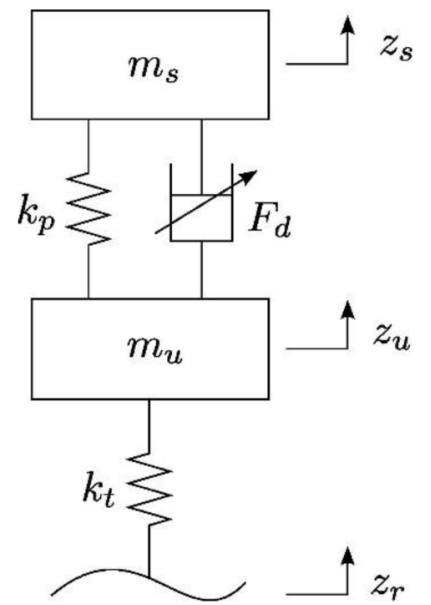

Figure 2. Quarter car model with a variable damping force

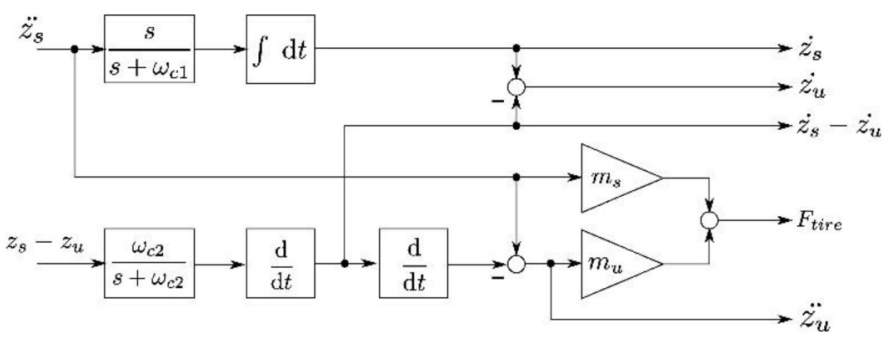

Figure 3. Estimator structure for various states of the system

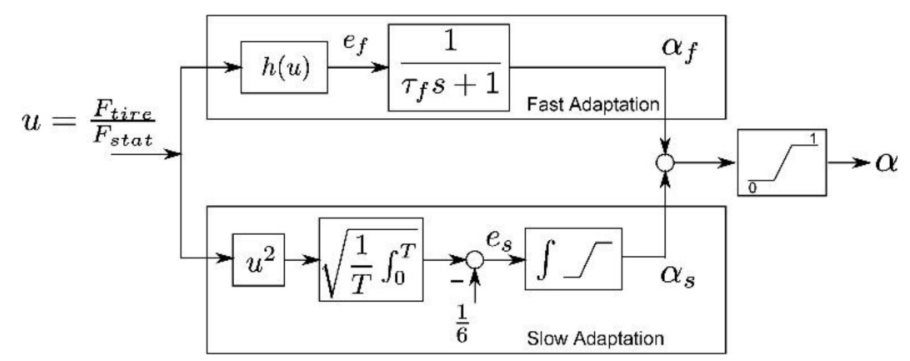

Figure 4. Structure of the adaptation logic to obtain scheduling parameter

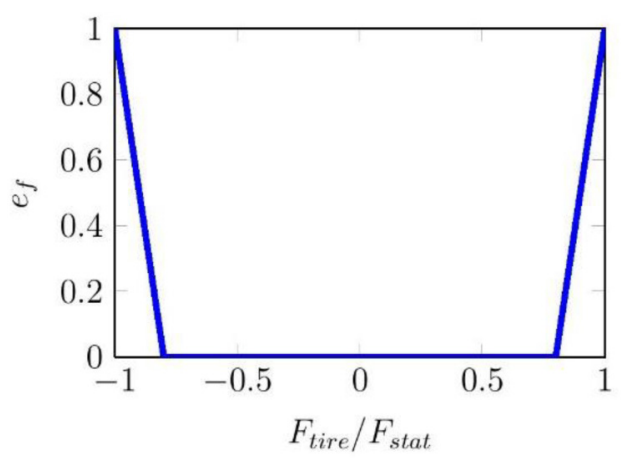

Figure 5. Heuristic function $h(u)$ for calculation of fast adaptation error $e_{f}$

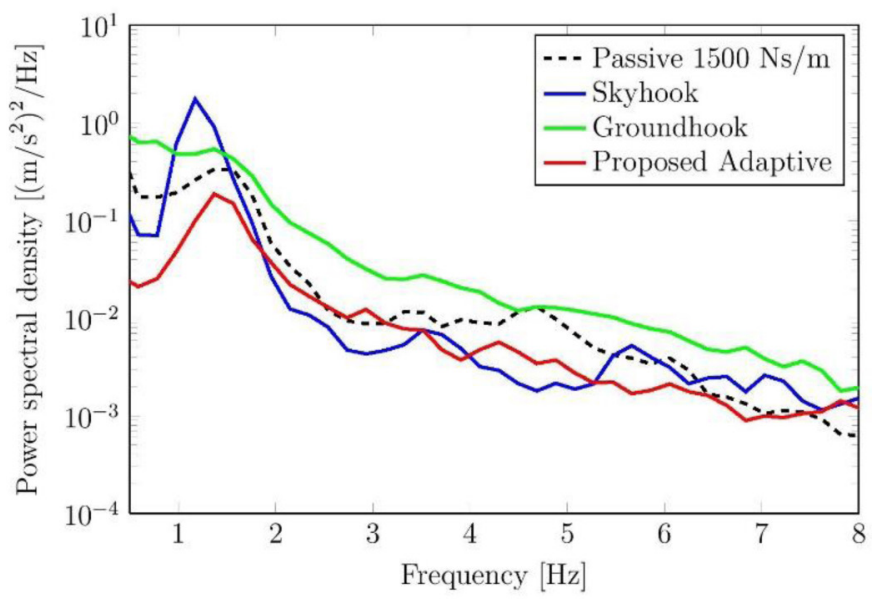

Figure 6. Simulation Results for a transition from type-A road to type-C road at $15 \mathrm{~m} / \mathrm{s}$ : Power spectral density of the acceleration of the sprung mass in the frequency band of maximum human sensitivity

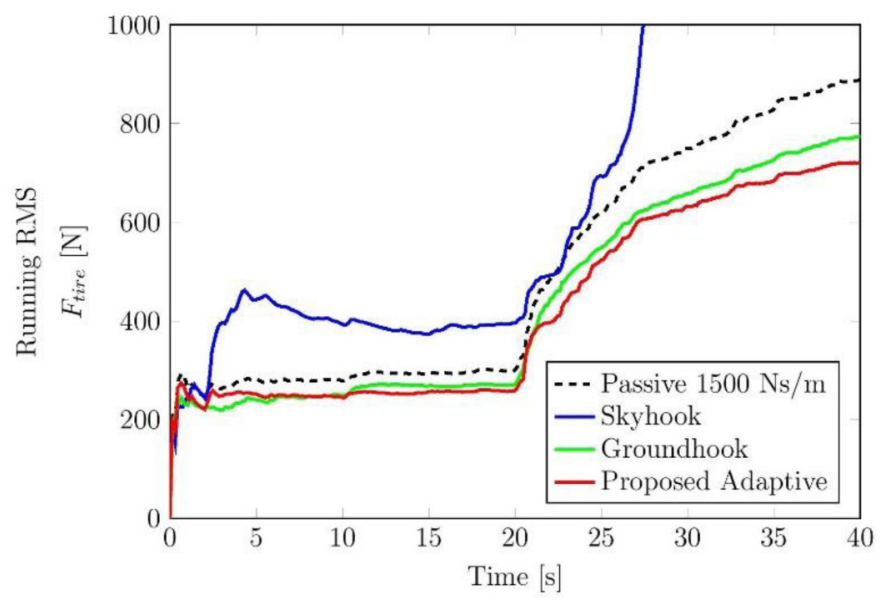

Figure 7. Simulation Results for a transition from type-A road to type-C road at $15 \mathrm{~m} / \mathrm{s}$ : Running RMS of the dynamic tire force

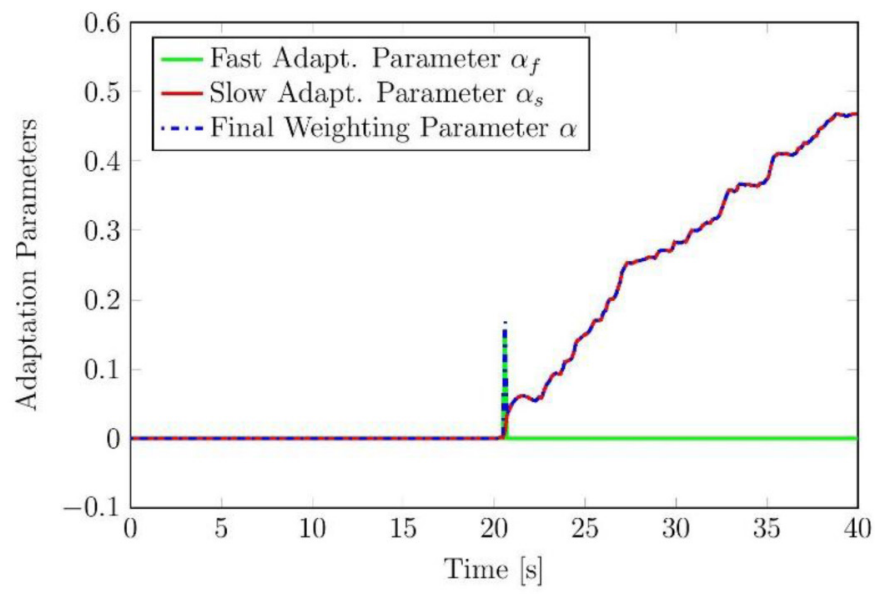

Figure 8. Simulation Results for a transition from type-A road to type-C road at $15 \mathrm{~m} / \mathrm{s}$ : Variation of adaptation parameters with time 


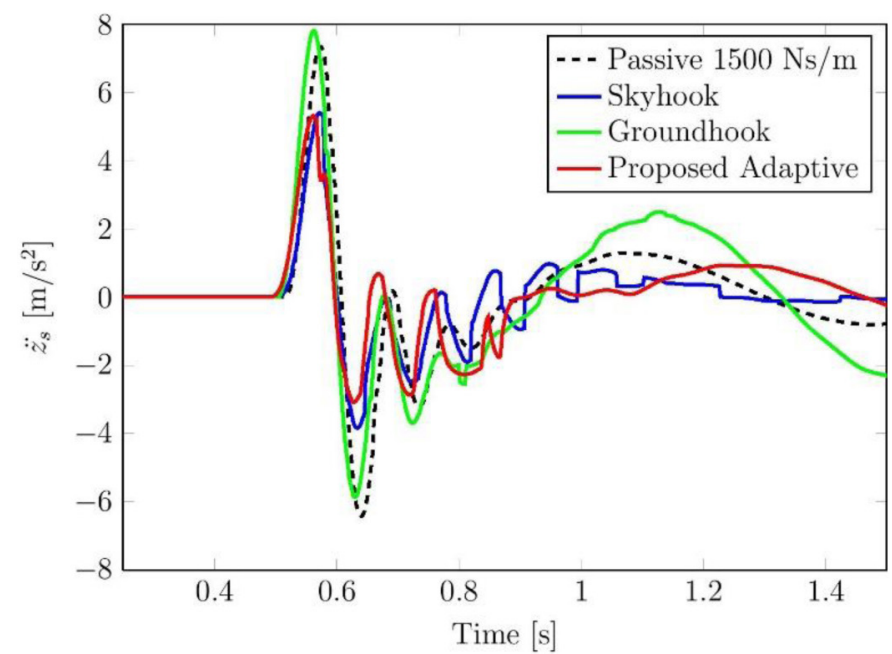

Figure 9. Simulation results for over a bump at speed $10 \mathrm{~m} / \mathrm{s}$ : Variation of acceleration of the sprung mass with time

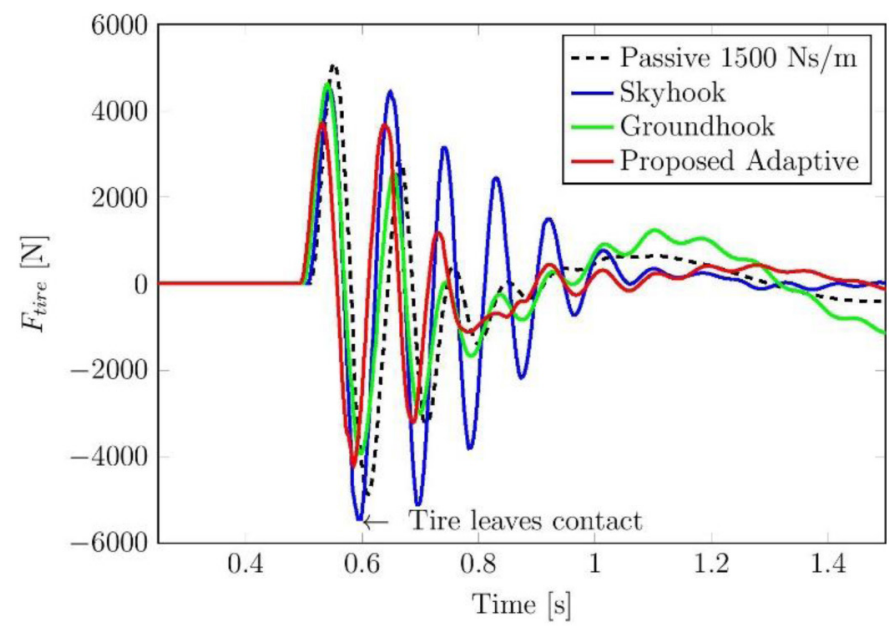

Figure 10. Simulation results for over a bump at speed $10 \mathrm{~m} / \mathrm{s}$ : Variation of dynamic tire force with time

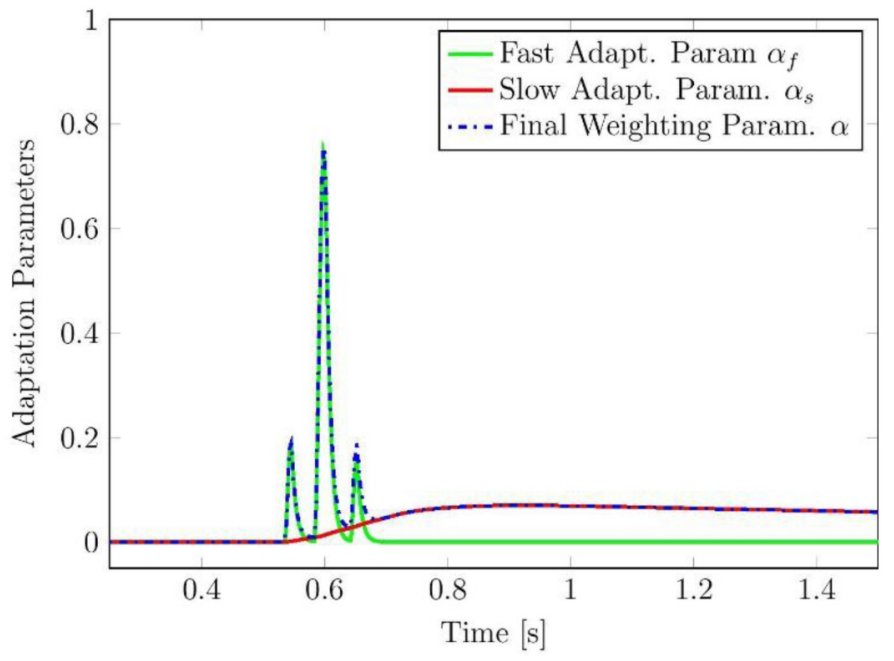

Figure 11. Simulation results for over a bump at speed $10 \mathrm{~m} / \mathrm{s}$ :

Variation of adaptation parameters with time

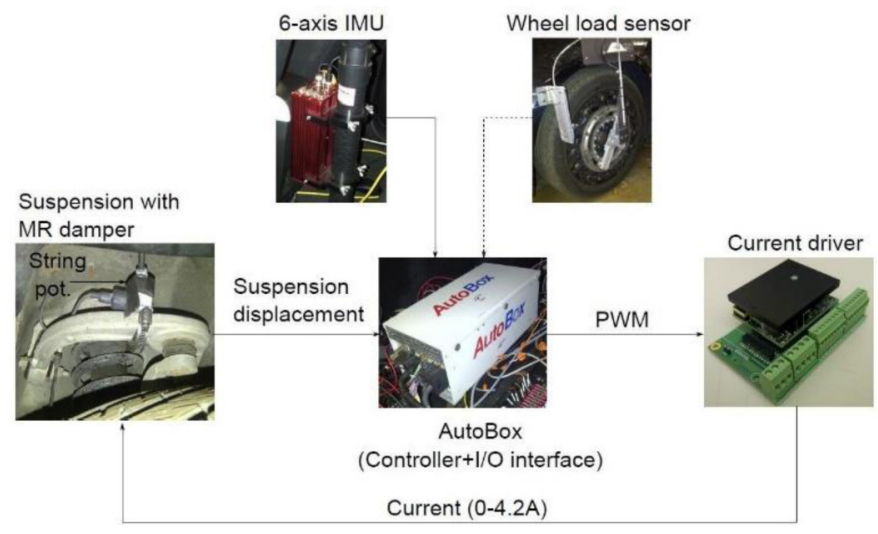

Figure 12. Structure of the experimental setup

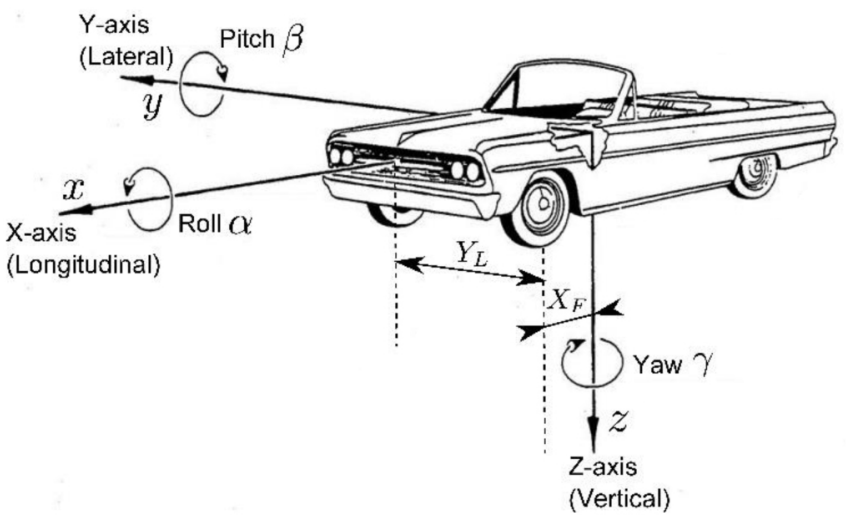

Figure 13. SAE Coordinate system for vehicle dynamics. Image adapted from [13] and then modified

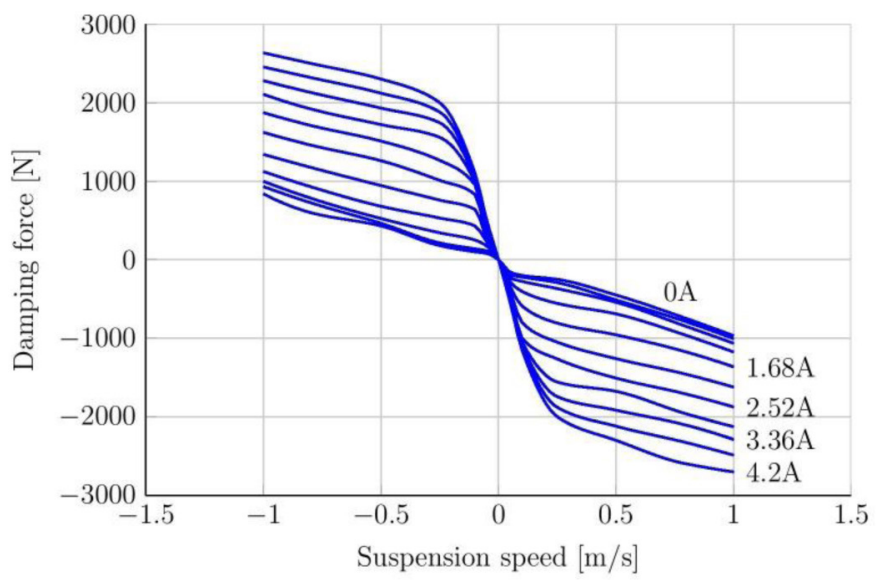

Figure 14. Characteristics of the MR damper (obtained after a smooth curve-fit) showing damping force as a function of suspension velocity at different currents 


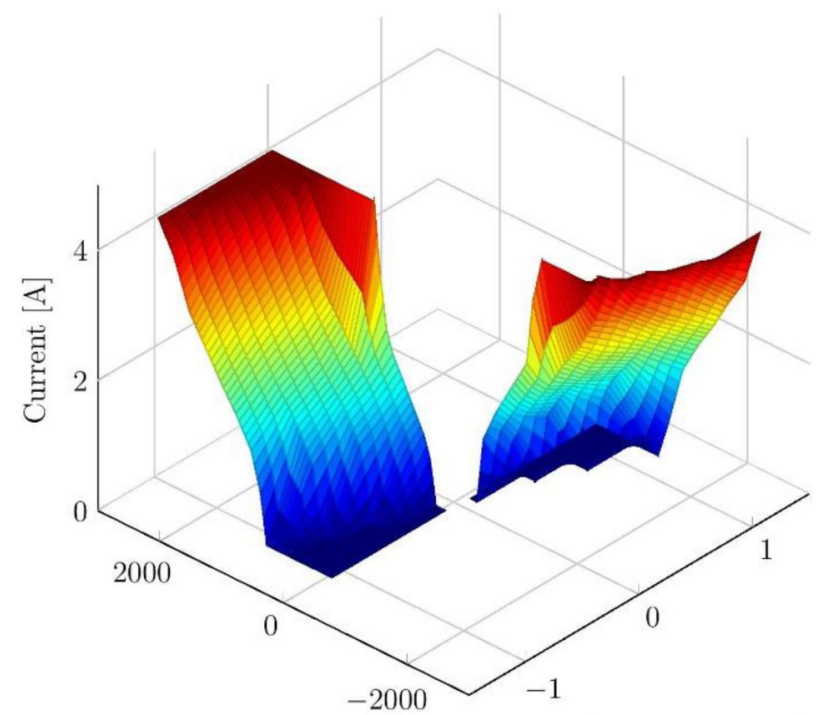

Damping force $[\mathrm{N}]$

Suspension speed $[\mathrm{m} / \mathrm{s}]$

Figure 15. Inverse mapping from MR damper characteristics

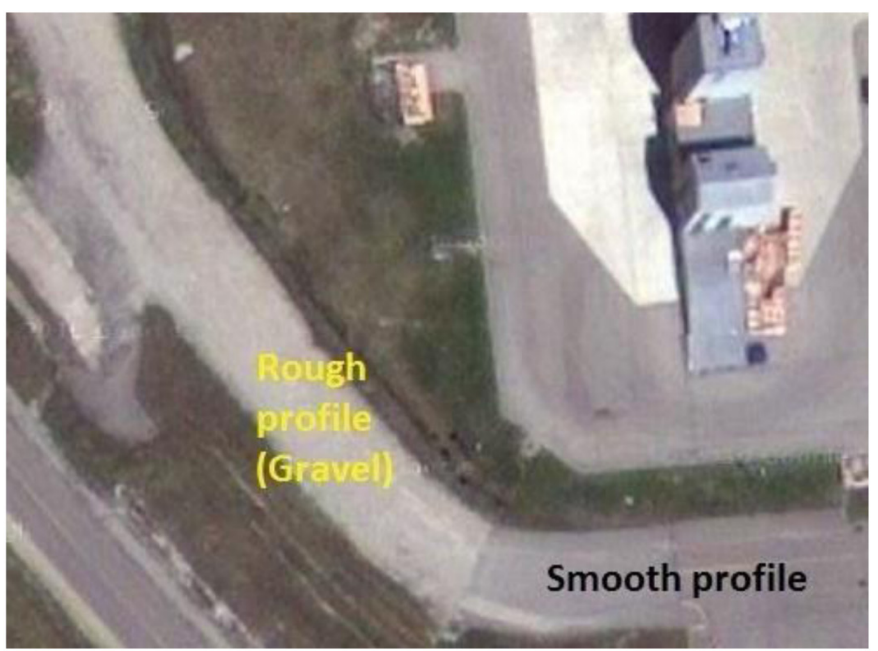

Figure 16. Satellite image of the test track showing smooth and rough profiles

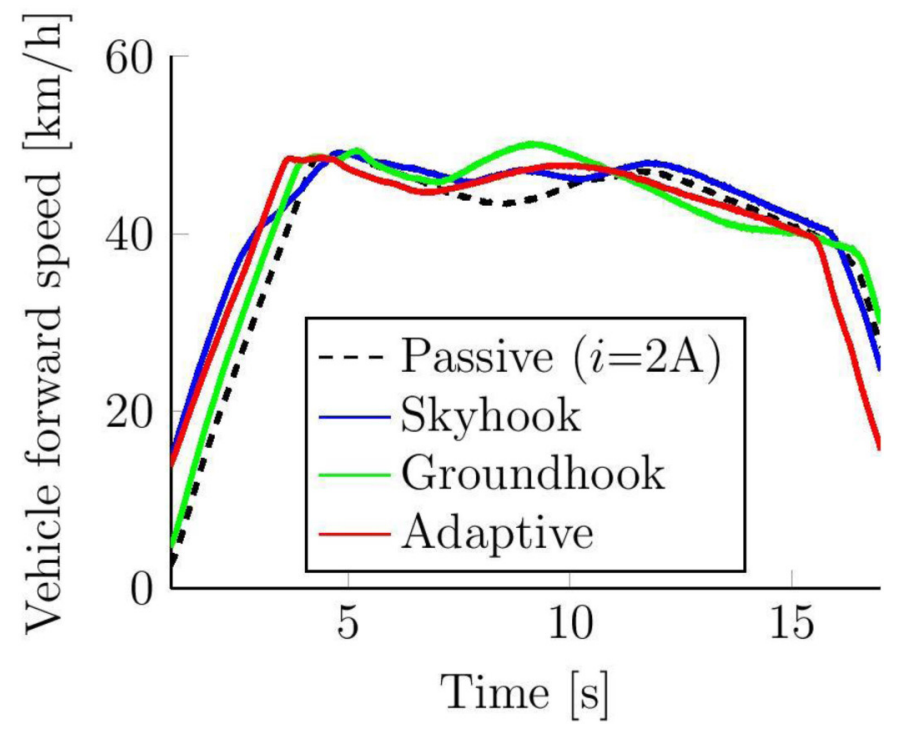

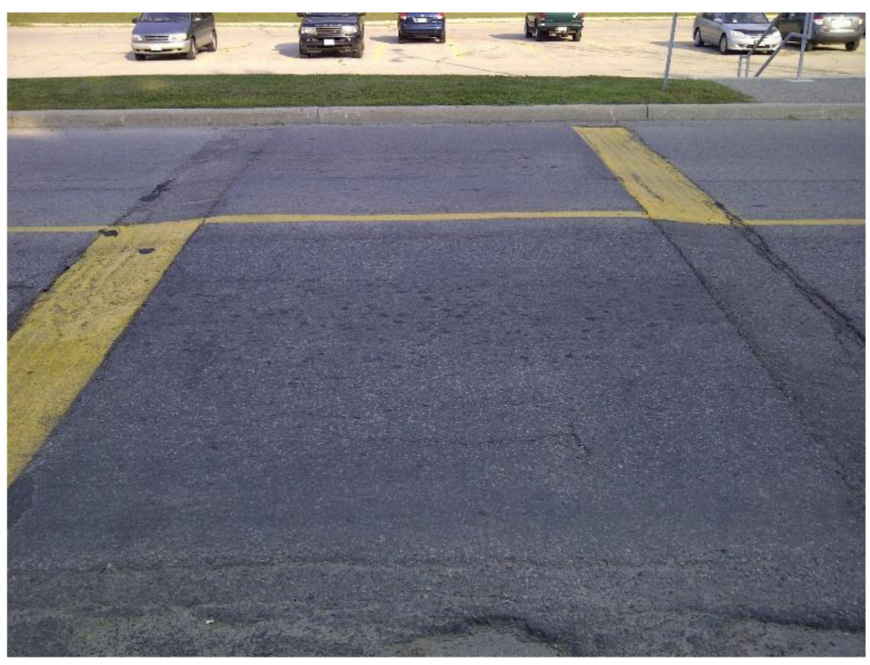

Figure 18. Image of the bump over which vehicle was tested

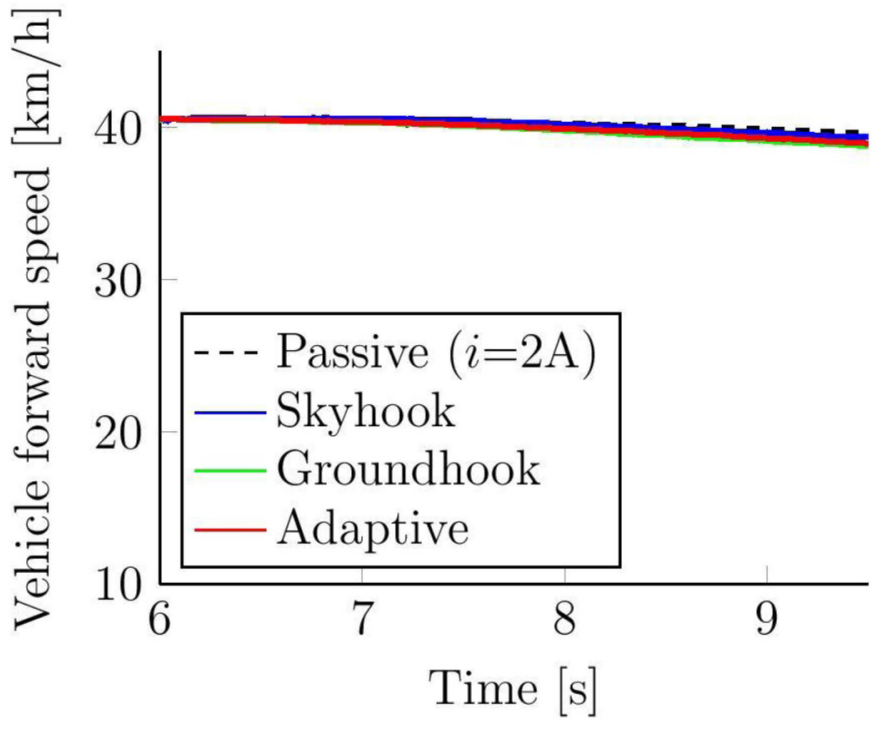

Figure 19. Forward velocity of the vehicle for the four runs on bump

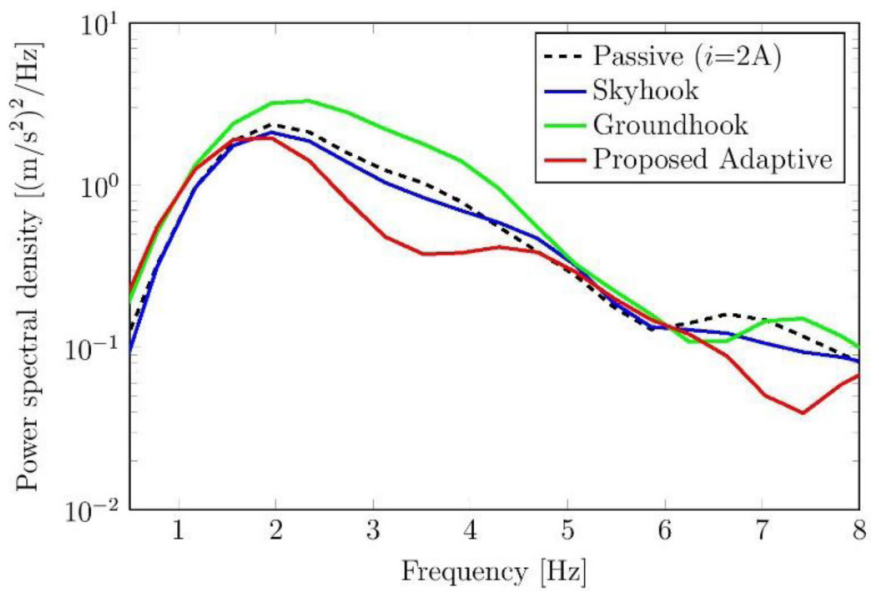

Figure 20. Test results for a transition from smooth to rough (gravel) road at $45 \mathrm{~km} / \mathrm{h}$ : PSD of the acceleration of the corner 


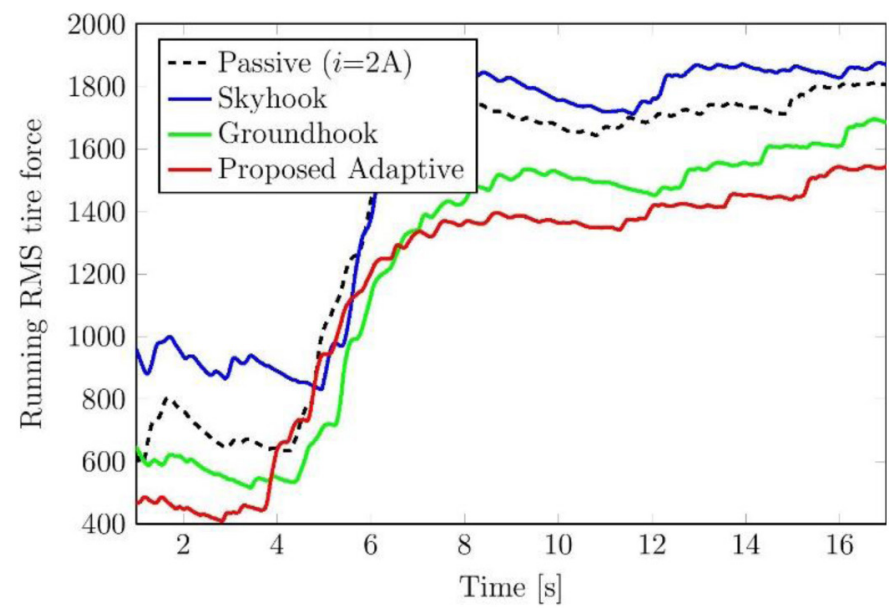

Figure 21. Test results for a transition from smooth to rough (gravel) road at $45 \mathrm{~km} / \mathrm{h}$ : Running RMS of the dynamic tire force

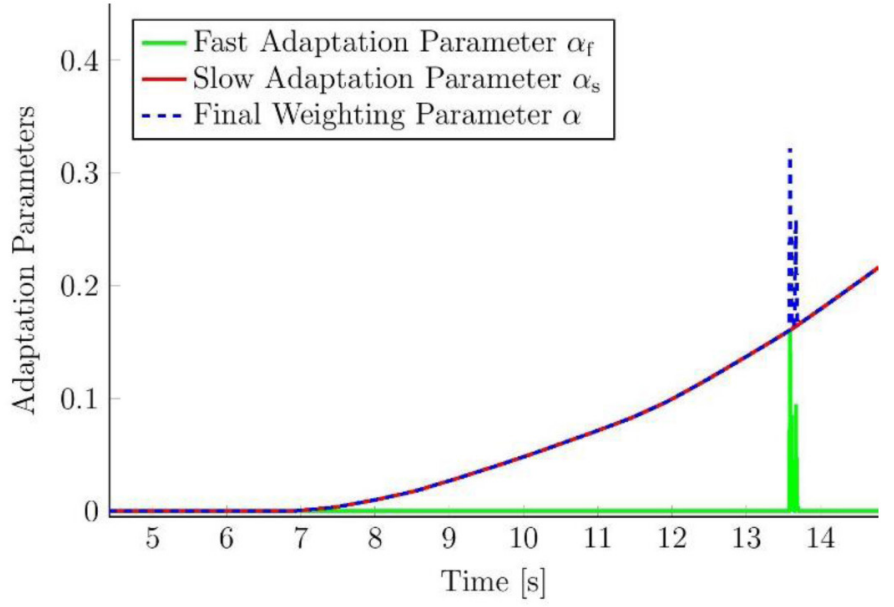

Figure 22. Test results for a transition from smooth to rough (gravel) road at $45 \mathrm{~km} / \mathrm{h}$ : Variation of adaptation parameters with time

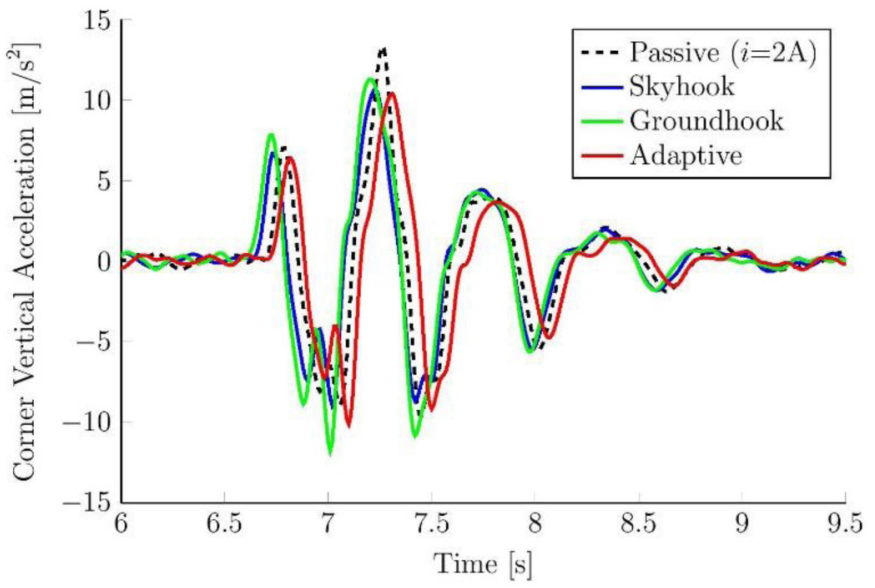

Figure 23. Test results for over a bump at speed $40 \mathrm{~km} / \mathrm{h}$ : Variation of acceleration of the corner with time

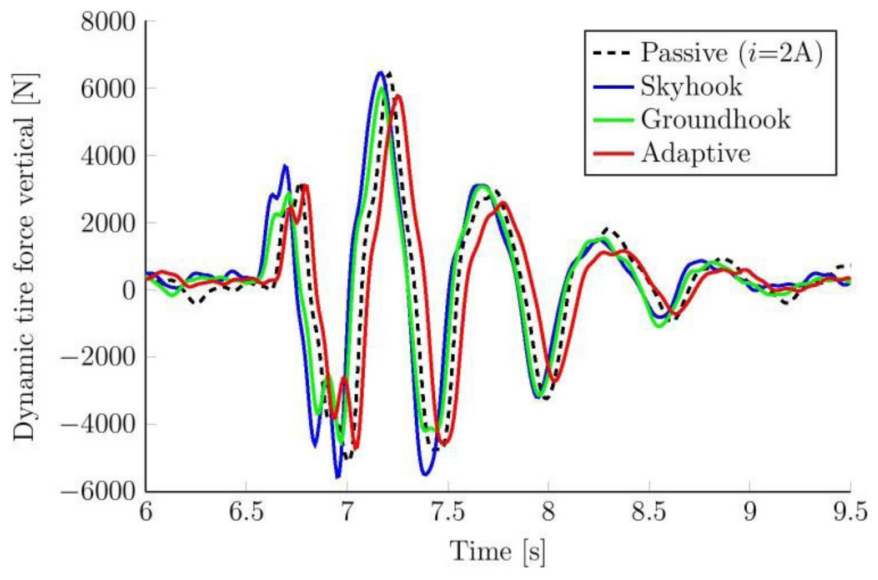

Figure 24. Test results for over a bump at speed $40 \mathrm{~km} / \mathrm{h}$ : Variation of dynamic tire force with time

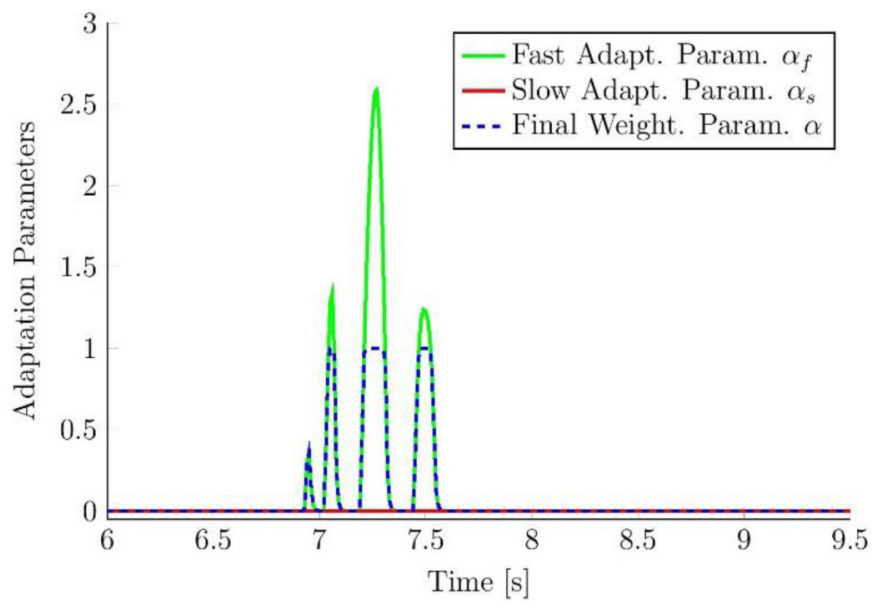

Figure 25. Test results for over a bump at speed $40 \mathrm{~km} / \mathrm{h}$ : Variation of adaptation parameters with time

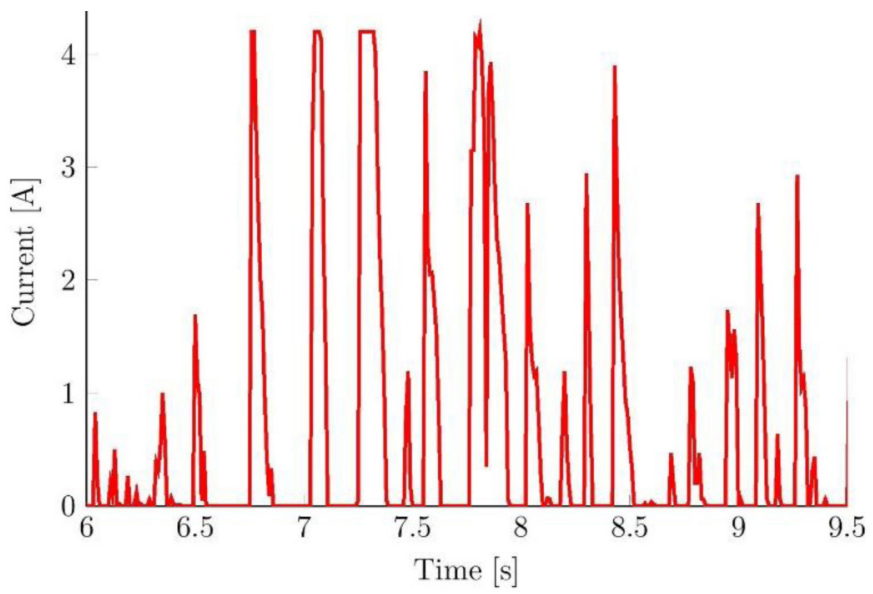

Figure 26. Test results for over a bump at speed $40 \mathrm{~km} / \mathrm{h}$ : Variation of adaptation parameters with time 


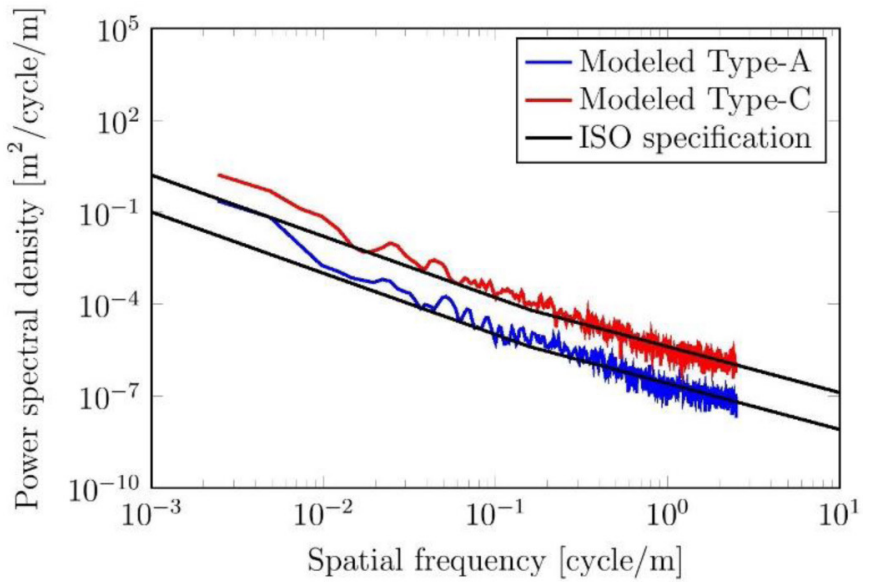

Figure A1. Power spectral density of two modeled road profiles with their ISO specified counterpart

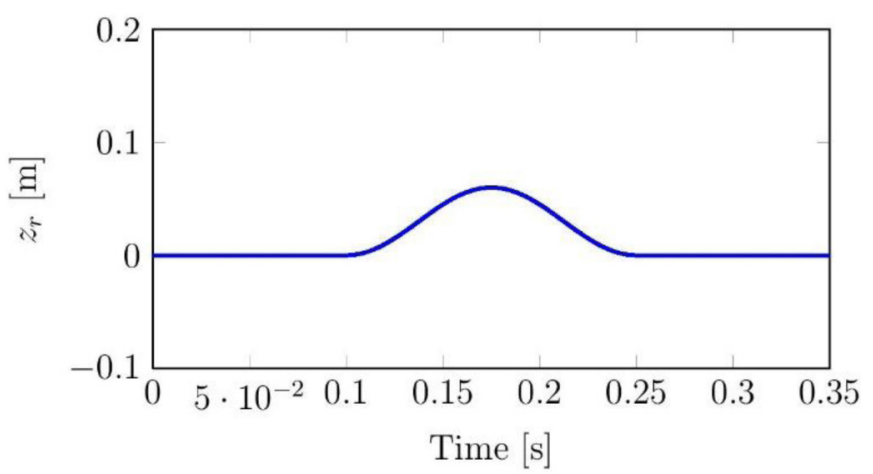

Figure B1. Model of a road bump as a function of time 


\section{APPENDIX}

\section{APPENDIX A: ISO8608 SPECIFICATION FOR ROAD PROFILES}

A road profile can be approximately modeled in time domain as constant $K$ times integrated white noise. The constant parameter is assigned such that the power spectral density of the modeled signal closely resembles with that of the ISO8608 specification [14] as shown in Figure A1.

$$
z_{r}(s)=\frac{K}{s} w(s)
$$

where $w(s)$ is white noise signal.

\section{APPENDIX B: MODELING A BUMP}

A simple model for a bump can be [15]

$$
z_{r}= \begin{cases}h_{b}\left(1-\cos \left(\frac{2 \pi V\left(t-t_{0}\right)}{L_{b}}\right)\right) & \text { for } t_{0} \leq t \leq \frac{L_{b}}{V}+t_{0} \\ 0 & \text { otherwise }\end{cases}
$$

where $h_{b}$ is half the bump height, $L_{b}$ is the length of the bump and $t_{0}$ is the time instant when the bump starts. For a vehicle speed of $10 \mathrm{~m} / \mathrm{s}$, an exemplary bump model with height $6 \mathrm{~cm}$ is shown in Figure B1.

All rights reserved. No part of this publication may be reproduced, stored in a retrieval system, or transmitted, in any form or by any means, electronic, mechanical, photocopying, recording, or otherwise, without the prior written permission of SAE International.

Positions and opinions advanced in this paper are those of the author(s) and not necessarily those of SAE International. The author is solely responsible for the content of the paper. 